\title{
Temperature dependence of the superconductivity-induced collective mode in the $c$-axis infrared spectra of bilayer cuprate superconductors
}

\author{
D. Munzar ${ }^{\mathrm{a}, *}$, J. Chaloupka $^{\mathrm{a}}$, C. Bernhard ${ }^{\mathrm{b}}$, A. Dubroka ${ }^{\mathrm{b}}$, J. Vašátko $^{\mathrm{a}}$ \\ a Department of Condensed Matter Physics, Faculty of Science, Masaryk University, Kotlářská 2, 61137 Brno, Czech Republic \\ ${ }^{\mathrm{b}}$ Department of Physics and Fribourg Center for Nanomaterials, Chemin du Musee 3, CH-1700 Fribourg, Switzerland
}

\begin{abstract}
The low-temperature spectra of the $c$-axis infrared conductivity of bilayer high- $T_{c}$ cuprate superconductors (HTCS) exhibit two superconductivity-induced modes [Li Yu et al., Phys. Rev. Lett. 100 (2008) 177004; and references therein]. Both can be understood in terms of a microscopic theory developed recently [J. Chaloupka, C. Bernhard, D. Munzar, Phys. Rev. B 79 (2009) 184513]. Here we summarize the elements of the theory and report on the temperature dependence (TD) of the low-energy mode and of the total optical spectral weight (SW). The calculated TD of the mode is consistent with experiment but the trends of the SW are not.
\end{abstract}

The important issue of the origin of the superconductivity-induced modes in the spectra of the $c$-axis infrared response of bilayer HTCS has been recently addressed using a microscopic theory [1]. Its elements are: (a) the local (intra-bilayer - "bl" and inter-bilayer - "int") fields, current densities, conductivities, and an extension of the multilayer formula of Ref. [2]. (b) The conductivities are calculated using a microscopic model and the linear response theory. This is the main difference with respect to previous phenomenological approaches [2-4]. (c) The microscopic model involves the bilayer-split (bonding and antibonding) bands. (d) The quasiparticles of the two bands are coupled to spin fluctuations, as in Ref. [5]. The coupling leads to superconductivity and is described at the level of generalized Eliashberg theory, as in Ref. [6]. (e) The gauge invariance of the theory, required for a consistent, i.e., charge conserving description of the charging of the copper-oxygen planes induced by the applied field, has been achieved by including a class of vertex corrections (VC) ensuring that the renormalized current vertices satisfy the appropriate Ward identities. The VC lead to dramatic and qualitative changes of the calculated response as demonstrated below. Fig. 1 shows the real part of the intra-bilayer conductivity calculated assuming insulating spacing layers between the bilayer units, considering a BCS-like (separable, nonretarded) interaction between the quasiparticles, for various values of the intrabilayer hopping amplitude $t_{\perp}$ (the values of other input parameters are the same as in Section III A of Ref.

\footnotetext{
* Corresponding author. Tel.: +420 549495980; fax: +420 541211214 .

E-mail address: munzar@physics.muni.cz (D. Munzar).
}

[1]), with the VC neglected (a) and included (b). In the former case, the conductivity possesses also the delta function at the origin due to the condensate (not shown). Note the difference in the $t_{\perp}$ dependence of the energy of the maximum: in (a), it begins at the superconducting gap $2 \Delta$, in (b), the energy is proportional to $t_{\perp}$. The maxima correspond to a pair-breaking (bonding-antibonding) peak and a collective mode (CM), respectively. As discussed in [1], the CM can be viewed as an analogue of the Bogoljubov-Anderson mode occurring in the longitudinal response of homogeneous superconductors, see Fig. 1c and d. For small values of the bilayer splitting, its nature is similar to that of the transverse plasmon introduced in Ref. [2]. The $t_{\perp}$-dependence of its frequency is analogous to the $v_{F}$ (Fermi velocity)-dependence of that of the Bogoljubov-Anderson mode, $\omega \sim v_{F}|\mathbf{q}|$, where $\mathbf{q}$ is the wavevector. In the total $c$-axis conductivity, $\sigma_{c}$, the $\mathrm{CM}$ is shifted towards higher energies due to the Coulomb interaction of the charged planes, an analogue of the Anderson-Higgs mechanism. The interpretation of the two modes in the data is the following: the well known peak around $400 \mathrm{~cm}^{-1}$ is attributed to the $\mathrm{CM}$ and the maximum around $1000 \mathrm{~cm}^{-1}$ [7] to a pair-breaking feature resulting from the coupling through the spacing layers. The theory can be further extended [8] to account for the phonon anomalies discussed in Ref. [3]. The resulting formulas have the same form as those of Refs. [3,9].

Fig. 2 shows the TD of the CM in $\operatorname{Re} \sigma_{c}$ calculated assuming insulating spacing layers, quasiparticles coupled to spin fluctuations, VC included, $t_{\perp \max }=45 \mathrm{meV}$, the values of other input parameters are the same as in Section III B of Ref. [1]. Above $T_{c}$ of $89 \mathrm{~K}$, the spectra display a broad maximum centered around $600 \mathrm{~cm}^{-1}$ due 

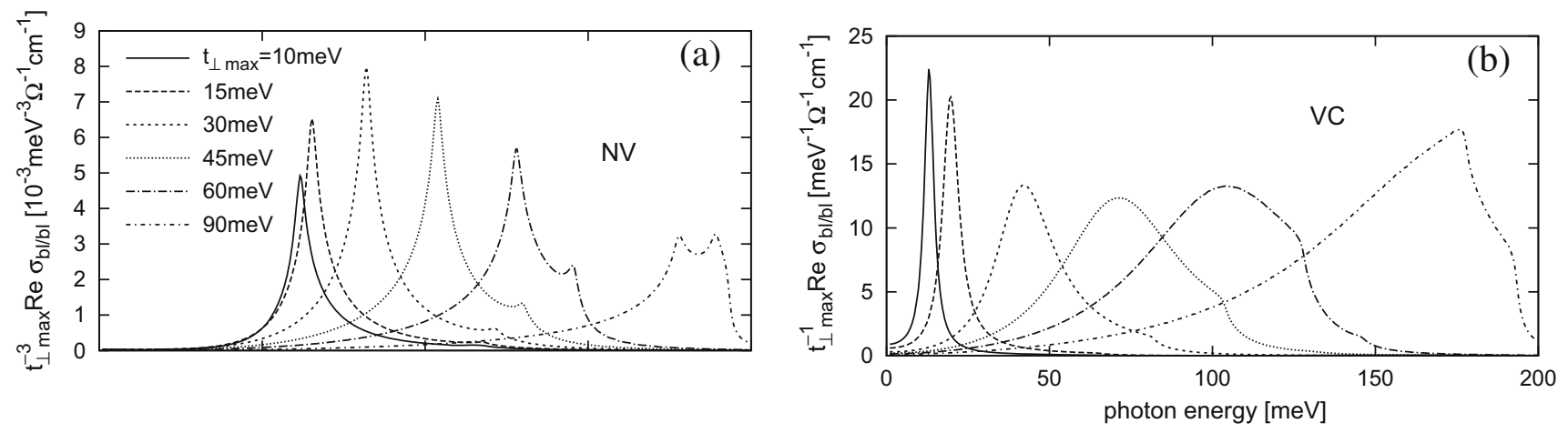

(c)

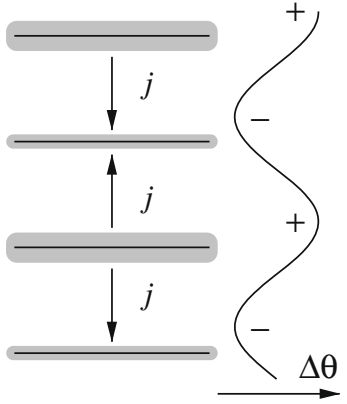

(d)

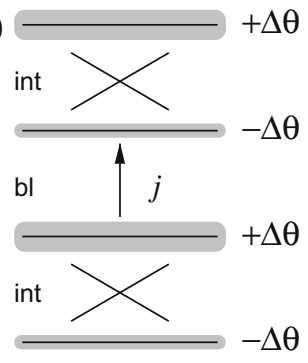

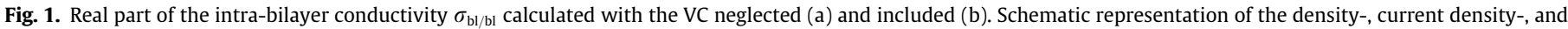
phase-pattern associated with the Bogoljubov-Anderson mode of a single layer superconductor (c) and the same for the collective mode of the bilayer system (d).

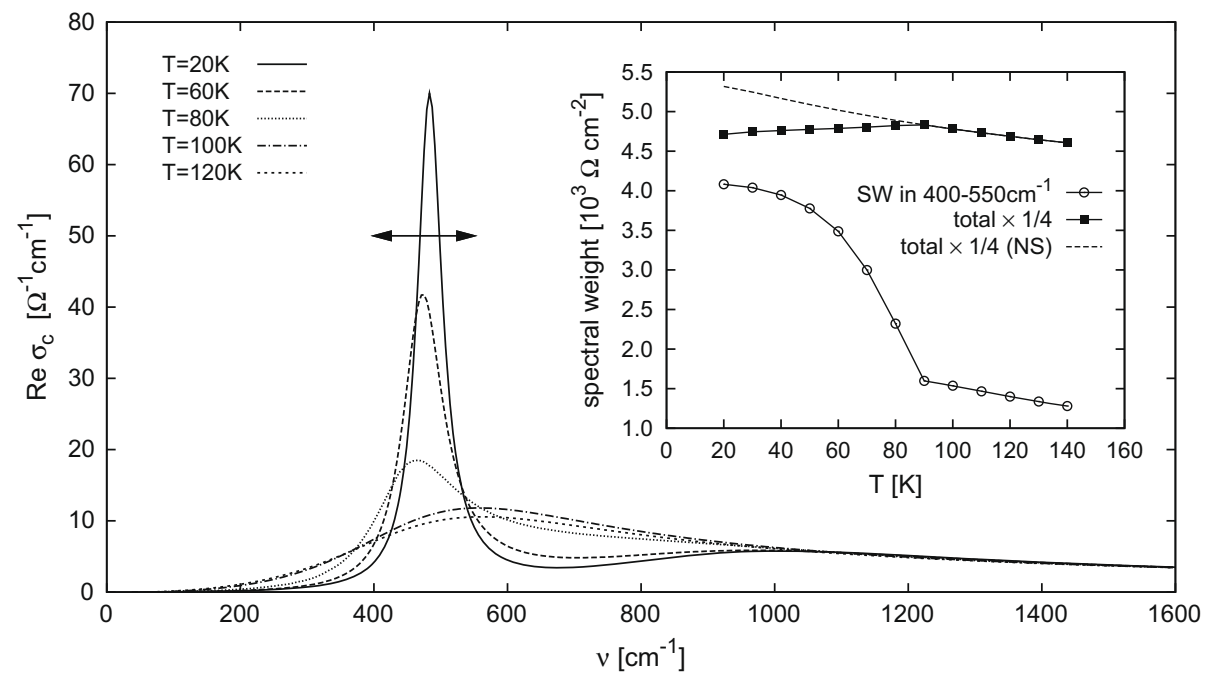

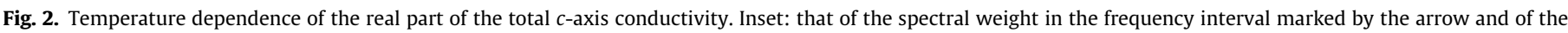
total optical spectral weight. The dashed line represents a straightforward extrapolation of the normal state dependence.

to the interband transitions. Only below $T_{c}$, a sharp peak corresponding to the CM appears around $480 \mathrm{~cm}^{-1}$, collecting SW both from lower and from higher energies. Its frequency is only weakly temperature dependent, in agreement with the experimental data on underdoped $\mathrm{YBa}_{2} \mathrm{Cu}_{3} \mathrm{O}_{7-\delta}$ [10]. Its $\mathrm{SW}$ exhibits an order-parameter like increase below $T_{c}$, in agreement with the data on slightly underdoped samples [10]. The total optical SW exhibits a decrease below $T_{c}$. This is consistent with the BCS theory and in contrast with the observed increase of the SW in the infrared [11,12,7]. A slightly different temperature dependence of the total SW would be obtained in case of a temperature dependent spin susceptibility $\chi_{\mathrm{SF}}$. The total SW decreases considerably with decreasing frequency of the low-energy component of $\chi_{\mathrm{sF}}$. The shift from
$40 \mathrm{meV}$ to $30 \mathrm{meV}$, e.g., leads to a decrease of the normal-state SW by about $5 \%$.

In conclusion, the calculated frequency of the collective mode is only weakly temperature dependent and its SW exhibits an order-parameter like behaviour, consistent with experiment. The TD of the total optical SW, however, displays a decrease below $T_{c}$, in contrast with experiment. The discrepancy is probably related to the inadequacy of the present approach to describe the $c$-axis electrodynamics in the normal state. Alternatively, it may be due to a strong TD of the pairing "glue". This work was supported by the Ministry of Education of Czech Republic (Grant No. MSM0021622410) and the Schweizerische Nationalfonds (SNF) (Grant No. 200020-119784). 


\section{References}

[1] J. Chaloupka, C. Bernhard, D. Munzar, Phys. Rev. B 79 (2009) 184513.

[2] D. van der Marel, A. Tsvetkov, Czech J. Phys. 46 (1996) 3165.

[3] D. Munzar et al., Solid State Commun. 112 (1999) 365.

[4] M. Grüninger et al., Phys. Rev. Lett. 84 (2000) 1575.

[5] M. Eschrig, M.R. Norman, Phys. Rev. Lett. 89 (2002) 277005.
[6] J. Chaloupka, D. Munzar, Phys. Rev. B 76 (2007) 214502.

[7] Li Yu et al., Phys. Rev. Lett. 100 (2008) 177004

[8] J. Vašatko et al., unpublished data.

[9] A. Dubroka, D. Munzar, Physica C 405 (2004) 133

[10] C. Bernhard et al., Phys. Rev. B 61 (2000) 618

[11] D.N. Basov et al., Science 283 (1999) 49.

[12] A.V. Boris et al., Phys. Rev. Lett. 89 (2002) 277001. 